\title{
Resonant Control of Elastic Collisions in an Optically Trapped Fermi Gas of Atoms
}

\author{
T. Loftust, C. A. Regal, C. Ticknor, J. L. Bohn, and D. S. Jint \\ JILA, National Institute of Standards and Technology and University of Colorado, Boulder, Colorado 80309
}

(29 November, 2001)

\begin{abstract}
We have loaded an ultracold gas of fermionic atoms into a far off resonance optical dipole trap and precisely controlled the spin composition of the trapped gas. We have measured a magnetic-field Feshbach resonance between atoms in the two lowest energy spin-states, $|9 / 2,-9 / 2\rangle$ and $|9 / 2,-7 / 2\rangle$. The resonance peaks at a magnetic field of $201.5 \pm 1.4 \mathrm{G}$ and has a width of $8.0 \pm 1.1 \mathrm{G}$. Using this resonance we have changed the elastic collision cross section in the gas by nearly 3 orders of magnitude.
\end{abstract}

PACS number(s): 34.50.-s, 32.80.Pj, 05.30.Fk

Magnetic-field Feshbach resonances have recently emerged as a powerful tool for studies of ultracold and quantum degenerate gases 11,4 . These resonances arise when the collision energy of two free atoms coincides with that of a quasi-bound molecular state [5,66. To access the resonance a magnetic field is applied to tune relative energies through the Zeeman effect. Measurements of Feshbach resonances enable highly accurate determinations of molecular interaction parameters [3, ㄱ, 8]. More importantly, a Feshbach resonance gives the experimenter the unique ability to control the interaction strength in a system. The interactions between atoms in a gas can be adjusted from very weak to very strong simply by controlling the magnitude of an applied magnetic field. In addition, a Feshbach resonance can be used to control the sign of the s-wave scattering length, which determines whether interactions between atoms are effectively repulsive or attractive. These abilities were recently exploited in ${ }^{85} \mathrm{Rb}$ experiments where a Feshbach resonance was used to facilitate evaporative cooling to Bose-Einstein condensation [9]. Furthermore, the resonance enabled a detailed study of the collapse dynamics of Bose-Einstein condensates with attractive interactions [10]. In a quantum degenerate Fermi gas of atoms 11 13] a magnetic-field Feshbach resonance has the potential to play a similar and equally valuable role in exploring radically different interaction regimes. Additionally, a Feshbach resonance in a Fermi gas offers the opportunity to access a predicted phase transition to a novel superfluid state 14 16].

In this Letter we report the observation of a Feshbach resonance between optically trapped fermionic atoms in two different internal states. An optical dipole trap was used to confine atoms in the two lowest energy Zeeman states of ${ }^{40} \mathrm{~K},\left|F=9 / 2, m_{F}=-9 / 2\right\rangle$ and $|9 / 2,-7 / 2\rangle$, where $F$ is the total atomic spin and $m_{F}$ is the magnetic quantum number. The Feshbach resonance between atoms in these states drives a dramatic change in the measured cross-dimensional rethermalization rate, with a peak at an applied magnetic field of $201.5 \pm 1.4 \mathrm{G}$, in agreement with the theoretical prediction in Ref. 17].

The fermionic isotope ${ }^{40} \mathrm{~K}$, with a nuclear spin of 4 , has a wealth of spin-states available for study (see Fig. 1). The procedure for creating an ultracold gas of ${ }^{40} \mathrm{~K}$ atoms builds upon our previous cooling and trapping techniques [11, 18. Atoms in the $|9 / 2,9 / 2\rangle$ and $|9 / 2,7 / 2\rangle$ states are first held in a magnetic trap and cooled by forced evaporation. (Two spin components are used for the evaporative cooling because fermions in the same internal state stop colliding at ultralow temperatures 19].) The ultracold gas is then loaded into a far-off resonance optical dipole trap (FORT). The FORT provides additional experimental capabilities, such as the ability to confine atoms in any combination of hyperfine spin-states with a trapping potential that is independent of an atom's magnetic moment [22]. This is particularly useful when working with fermionic atoms since the realization of an interacting quantum Fermi gas requires two (or more) components 18,20,21]. In addition, optical trapping allows for the application of arbitrary magnetic fields. Both capabilities are essential for accessing the predicted Feshbach resonance in ${ }^{40} \mathrm{~K}$ since it occurs for collisions between atoms in the "strong-field-seeking" $|9 / 2,-9 / 2\rangle$ and $|9 / 2,-7 / 2\rangle$ states, which cannot be confined in a static magnetic trap.

The FORT consists of a single focused beam obtained from a $\lambda=1064 \mathrm{~nm}$ Nd:YAG laser. The trapping beam passes through an acousto-optic modulator, which is used for intensity stabilization and switching. The beam is spatially filtered with a single-mode optical fiber before being focused to a waist of approximately $20 \mu \mathrm{m}$ at the center of the magnetic trap. To load the atoms into the FORT we simply overlap the FORT beam with the magnetic trap. The FORT is switched on to $0.18 \mathrm{~W}$ in $40 \mathrm{~ms}$ with a linear ramp. The magnetic trap is then turned off quickly ( $<1 \mathrm{~ms}$ switching time) $10 \mathrm{~ms}$ later [23].

Before imaging the atom cloud, the FORT light is suddenly switched off and the gas is allowed to expand freely. Following an expansion time of typically $6 \mathrm{~ms}$ we take a picture of the gas using resonant absorption imaging. Number, temperature, and center-of-mass position of the gas are extracted from the images. As this imaging technique is destructive, all the measurements described here involved repeated cycles of the experiment. To observe the spin-composition of the gas, a magnetic field with a strong vertical gradient is applied during the expansion from the FORT [24,18]. Atoms in different spin states 
then separate spatially via the Stern-Gerlach effect. Figure 1 shows a resonant absorption image of a gas that contains a mixture of all 10 Zeeman states in the $F=9 / 2$ hyperfine ground state of ${ }^{40} \mathrm{~K}$.

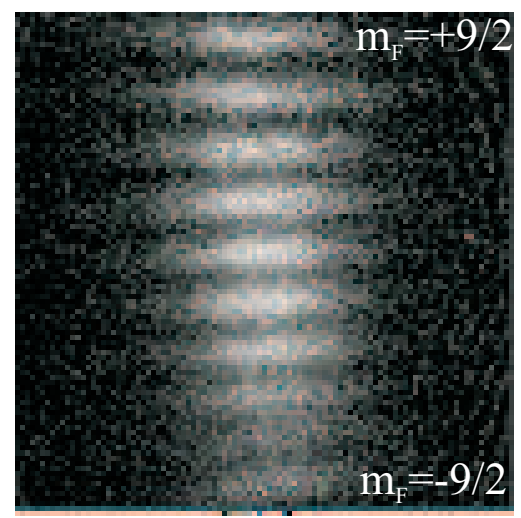

FIG. 1. A mixed spin-state gas released from the FORT. Atoms in the 10 Zeeman states of the ${ }^{40} \mathrm{~K} \mathrm{~F}=9 / 2$ ground state are simultaneously trapped in the FORT. Before imaging, atoms in different spin-states were separated with a Stern-Gerlach field applied during $7.3 \mathrm{~ms}$ of expansion from the FORT. This picture encompasses a $3.1 \mathrm{~mm}$ square area and is the sum of absorption images taken with $\sigma^{+}$and $\sigma^{-}$ light. The mixture of spin-states in the FORT was achieved by suddenly reversing the direction of an applied magnetic field.

To characterize the FORT strength, the radial frequency was measured by modulating the optical power and monitoring the resulting temperature of the gas. For a harmonic potential, parametric heating occurs when the modulation frequency equals twice the trap frequency. In parametric heating measurements we observed two transverse frequencies, vertical and horizontal, which differed by a factor of 1.6. We attribute this difference to astigmatism or asymmetry in the FORT beam optics. For the $0.8 \mathrm{~W}$ of optical power used for the measurements of the Feshbach resonance, the lower, vertical frequency, $\nu_{y}$, was $1.2 \mathrm{kHz}$. In the weaker axial direction the trap strength was measured by exciting centerof-mass motion with a pulsed magnetic field gradient. In the $0.8 \mathrm{~W}$ FORT the gas oscillated with a frequency of 15 $\mathrm{Hz}$. However, strong damping of this axial motion after only a couple cycles was indicative of strong anharmonicity in the axial potential. As an operational measure of the trap depth we found that the maximum temperature of a gas confined in the $0.8 \mathrm{~W}$ FORT was $18 \mu \mathrm{K}$.

Exploitation of a Feshbach resonance between atoms in two internal states requires precise control over the spin composition of the gas. Both elastic and inelastic collision rates can be strongly affected by spin composition impurity. For example, if the elastic collision crosssection between the two relevant states is tuned to zero using the Feshbach resonance, even a small fraction of atoms in a third spin state will dominate collisional in- teractions in the gas and set a finite elastic collision rate. In addition, atoms in other spin states can cause heating and number loss due to inelastic processes such as spin-exchange collisions.

For measurements of the Feshbach resonance, the spin composition of the gas was controlled using the following procedure. Prior to loading the FORT, the magnetically trapped ${ }^{40} \mathrm{~K}$ gas was spin-polarized with all the atoms in the $|9 / 2,9 / 2\rangle$ state. Atoms in the second spin-state used for the evaporative cooling, $|9 / 2,7 / 2\rangle$, were selectively removed with a frequency-swept microwave field, which drove transitions to an untrapped spin state in the upper hyperfine ground state. To ensure that the remaining gas contained only atoms in the $|9 / 2,9 / 2\rangle$ state, we applied a second frequency-swept microwave field that removed any atoms in the $|9 / 2,5 / 2\rangle$ and $|9 / 2,3 / 2\rangle$ states. The spin-polarized gas was then loaded into the FORT as described above. To preserve the spin composition we maintained an applied magnetic field of at least 2 gauss while the atoms were in the FORT.

After the gas is loaded into the FORT, we used adiabatic rapid passage to create a mixture of atoms in the $|9 / 2,-9 / 2\rangle$ and $|9 / 2,-7 / 2\rangle$ states. The transfer used an applied radio-frequency (rf) field and was performed in two steps. For both steps a static, spatially uniform 25] magnetic field of $28.5 \mathrm{G}$, generated by two of the magnetic trap coils, was applied along the weaker axial direction of the FORT. In the first step, the spinpolarized $|9 / 2,9 / 2\rangle$ gas was completely transferred to the $|9 / 2,-9 / 2\rangle$ state with a $10 \mathrm{~ms}$ long frequency sweep across all 9 Zeeman transitions. In the second step, approximately $40 \%$ of the atoms were transferred to the $|9 / 2,-7 / 2\rangle$ state by sweeping the rf frequency across the $|9 / 2,-9 / 2\rangle$ to $|9 / 2,-7 / 2\rangle$ transition in $20 \mathrm{~ms}$. With the rf sweeps optimized for minimal transfer to other Zeeman states, the fraction of atoms transferred to the $|9 / 2,-7 / 2\rangle$ spin state was $36 \pm 8 \%$.

For our measurements of the Feshbach resonance the FORT power was adiabatically increased from $0.18 \mathrm{~W}$ to $0.8 \mathrm{~W}$ and the magnetic field was increased to between $160 \mathrm{G}$ and $260 \mathrm{G}$. The elastic collision cross-section was then measured with a cross-dimensional rethermalization technique 26]. The cloud was taken out of thermal equilibrium by applying a parametric drive (modulating the optical trap power) to preferentially heat the gas in the vertical direction. The optical power in the FORT beam was modulated for 3 to $5 \mathrm{~ms}$ with a $30 \%$ amplitude and a frequency of $2 \nu_{y}$. Following parametric heating, thermal relaxation of the gas was observed in the time evolution of the rms cloud radii $z_{r m s}, y_{r m s}$ in the axial and radial directions (see Fig. 2). A rethermalization time constant $\tau$ was extracted from an exponential fit to the aspect ratio $z_{r m s} / y_{r m s}$ versus time. 


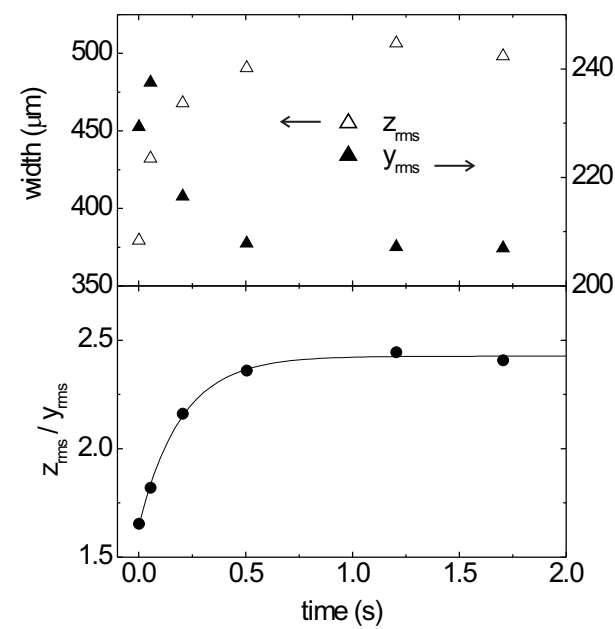

FIG. 2. Sample rethermalization data. The rethermalization of the optically trapped gas following modulation of the optical power is shown for a $212.6 \mathrm{G}$ magnetic field. The cloud widths $z_{r m s}$ and $y_{r m s}$ were obtained from gaussian fits to absorption images taken $6 \mathrm{~ms}$ after the gas was released from the FORT. Following the parametric heating, energy in the gas is redistributed by elastic collisions, and the ratio $z_{r m s} / y_{r m s}$ approaches its equilibrium value.

The elastic collision cross-section $\sigma$ was obtained from the measured rethermalization time $\tau$ using $\frac{1}{\tau}=\frac{2}{\alpha} n \sigma v$. The constant $\alpha=2.5$ is the calculated average number of binary $s$-wave collisions required for thermalization [19]. The mean relative speed is given by $v=4 \sqrt{\frac{k_{B} T}{\pi m}}$ and the density overlap by $n=\frac{1}{N} \int n(\vec{r})^{2} f(1-f) d^{3} \vec{r}$, where $f$ is the fraction of atoms in the $|9 / 2,-7 / 2\rangle$ spin-state. The total number of atoms $N$, the temperature $T$, and the density $n(\vec{r})$ were obtained from gaussian fits to absorption images of equilibrated samples.

Figure 3 shows the elastic collision cross-section $\sigma$ as a function of the applied magnetic field. The Feshbach resonance is clearly revealed as a change in $\sigma$ spanning nearly 3 orders of magnitude. At the peak of the resonance $\sigma$ is limited by the finite temperature of the gas ( $\mathrm{T}$ $=5 \mu \mathrm{K}$ ), while at the zero of the resonance $\sigma$ is limited by a background rethermalization rate. For the data shown in Fig. 3 we have subtracted this background rate, which was determined by measuring the rethermalization rate in a spin-polarized $|9 / 2,-9 / 2\rangle$ gas and is consistent with spin-state contamination at the level of $0.2 \%$. The error bars in Fig. 3 are dominated by uncertainties in $\tau$ and the background rate. In addition, $\sigma$ has an overall systematic uncertainty of approximately $\pm 50 \%$ that comes from measurements of $N$. The magnetic field values $B$ were calibrated using rf-driven spin-flip transitions and have a systematic uncertainty of $\pm 0.5 \%$.

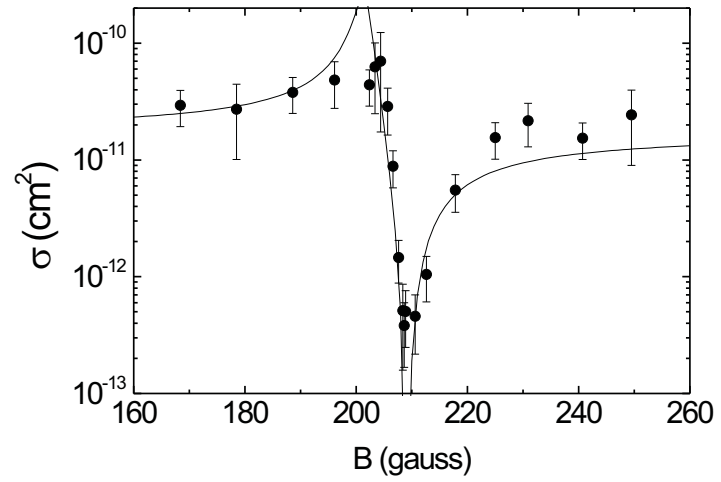

FIG. 3. Magnetic-field Feshbach resonance between ${ }^{40} \mathrm{~K}$ atoms in the $|9 / 2,-9 / 2\rangle$ and $|9 / 2,-7 / 2\rangle$ states. The measured elastic collision cross-section $\sigma$, obtained from measurements of the cross-dimensional rethermalization rate, is plotted as a function of applied magnetic field $B$. The data were taken with $N=8 \times 10^{6}, T=5 \mu \mathrm{K}$, and $f=0.36$. The solid line is a fit to the data from which we obtain cold collision parameters for potassium.

The solid line in Fig. 3 is the best-fit theory curve, computed from a multichannel scattering calculation whose details have been discussed elsewhere [27]. In this calculation the singlet and triplet scattering lengths $a_{s}$ and $a_{t}$ are regarded as fully independent fitting parameters that uniquely characterize the singlet and triplet potassium potentials. The model has been refined to incorporate both the accurate $a b$ initio van der Waals coefficient $C_{6}=3897 \pm 15$ [28] and the correct number of bound states in the ${ }^{39} \mathrm{~K}$ singlet and triplet potentials, $n_{s}=86$ and $n_{t}=27$, respectively [29]. For each value of applied magnetic field, thermally averaged cross sections are computed as described in Ref. 19. The reduced $\chi^{2}$ is then minimized with respect to $a_{s}, a_{t}$, and an overall scaling factor $\epsilon$ that accounts for the systematic uncertainty in the magnitude of the measured cross section. The resulting best fit, with $\chi^{2}=1.06$, yields $a_{s}=104.8 \pm 0.4$, $a_{t}=174 \pm 7$, and $\epsilon=1.30$. We find that $C_{6}$ does not play a critical role in the shape or the position of the resonance, but does affect the nominal value of $a_{t}$. This uncertainty is included in the quoted $a_{t}$ error bars. Performing the proper mass scaling, the scattering lengths of ${ }^{39} \mathrm{~K}$ are found to be $a_{s}=139.4 \pm 0.7 a_{o}$ and $a_{t}=-37 \pm 6 a_{0}$, where $a_{o}$ is the Bohr radius. These results are in agreement with the values of triplet scattering length reported in Ref. 29] $\left(a_{t}=-33 \pm 5 a_{o}\right)$ and the singlet scattering length reported in Ref. 27] $\left(a_{s}=140_{-6}^{+3} a_{o}\right)$.

In conclusion, we have demonstrated an optical trap for an ultracold gas of fermionic atoms. While the experiments described here were carried out in the nonde- 
generate regime (at approximately twice the Fermi temperature), future work will explore an optically trapped quantum degenerate gas. For example, with an appropriate choice of FORT and magnetic trap parameters it may be possible to achieve a high degree of degeneracy with an adiabatic compression of the Fermi gas [30]. Further, optical trapping has enabled us to observe a magnetic-field Feshbach resonance in ${ }^{40} \mathrm{~K}$. This resonance is one of only a handful of such resonances observed to date and furthermore is unique in that it occurs for fermionic atoms in different internal states. Future work will explore the utility of this tool for controlling collisional interactions in a dilute Fermi gas. We have already seen density dependent heating and number loss around the peak of the Feshbach resonance. Understanding these effects, presumably due to inelastic collisions, will be important for future applications. Of particular interest is the region where the s-wave scattering length is negative, between the peak and the minimum seen in our elastic collision cross-section measurements. Here the interparticle interactions are effectively attractive and could drive Cooper pairing of the fermionic atoms [14 16].

This work is supported by the National Science Foundation, the Office of Naval Research, and the National Institute of Standards and Technology. C. A. Regal acknowledges support from the Hertz Foundation.

* Quantum Physics Division, National Institute of Standards and Technology

[1] S. Inouye, M. R. Andrews, J. Stenger, H.-J. Miesner, D. M. Stamper-Kurn, and W. Ketterle, Nature (London) 392, 151 (1998).

[2] Ph. Courteille, R. S. Freeland, D. J. Heinzen, F. A. van Abeelen, and B. J. Verhaar, Phys. Rev. Lett. 81, 69 (1998).

[3] J. L. Roberts, N. R. Claussen, J. P. Burke, Jr., C. H. Greene, E. A. Cornell, and C. E. Wieman, Phys. Rev. Lett. 81, 5109 (1998).

[4] V. Vuletić, A. J. Kerman, C. Chin, and S. Chu, Phys. Rev. Lett. 82, 1406 (1999).

[5] W. C. Stwalley, Phys. Rev. Lett. 37, 1628 (1976).

[6] E. Tiesinga, A. J. Moerdijk, B. J. Verhaar, and H. T. C. Stoof, Phys. Rev. A 46, R1167 (1992); E. Tiesinga, B. J. Verhaar, and H. T. C. Stoof, Phys. Rev. A 47, 4114 (1993).

[7] J. L. Roberts, J. P. Burke, Jr., N. R. Claussen, S. L. Cornish, E. A. Donley, and C. E. Wieman, Phys. Rev. A 64, 024702 (2001).

[8] C. Chin, V. Vuletić, A. J. Kerman, and S. Chu, Phys. Rev. Lett. 85, 2717 (2000).

[9] S. L. Cornish, N. R. Claussen, J. L. Roberts, E. A. Cornell, and C. E. Wieman, Phys. Rev. Lett. 85, 1795 (2000).

[10] J. L. Roberts, N. R. Claussen, S. L. Cornish, E. A. Don- ley, E. A. Cornell, and C. E. Wieman, Phys. Rev. Lett. 86, 4211 (2001).

[11] B. DeMarco and D. S. Jin, Science 285, 1703 (1999).

[12] A. G Truscott, K. E. Strecker, W. I. McAlexander, G. B. Partridge, and R. G. Hulet, Science 291, 2570 (2001).

[13] F. Schreck, L. Khaykovich, K. L. Corwin, G. Ferrari, T. Bourdel, J. Cubizolles, and C. Salomon, Phys. Rev. Lett. 87, 80403 (2001).

[14] H. T. C. Stoof, M. Houbiers, C. A. Sackett, and R. G. Hulet, Phys. Rev. Lett. 76, 10 (1996).

[15] M. Holland, S. J. J. M. F. Kokkelmans, M. L. Chiofalo, and R. Walser, Phys. Rev. Lett. 87, 120406 (2001); M. L. Chiofalo, S. J. J. M. F. Kokkelmans, J. N. Milstein, and M. J. Holland, preprint cond-mat/0110119.

[16] E. Timmermans, K. Furuya, P. W. Milloni, and A. K. Kerman, Phys. Lett. A 285, 228 (2001).

[17] J. L. Bohn, Phys. Rev. A 61, 053409 (2000).

[18] B. DeMarco, S. B. Papp, and D. S. Jin, Phys. Rev. Lett. 86, 5409 (2001).

[19] B. DeMarco, J. L. Bohn, J. P. Burke, Jr., M. Holland, and D. S. Jin, Phys. Rev. Lett. 82, 4208 (1999).

[20] S. D. Gensemer and D. S. Jin, Phys. Rev.Lett. 87, 173201 (2001).

[21] B. DeMarco and D. S. Jin, cond-mat/0109098.

[22] J. Stenger, S. Inouye, M. R. Andrews, H.-J. Miesner, D. M. Stamper-Kurn, and W. Ketterle, Phys. Rev. Lett. 82, 2422 (1999).

[23] We have observed additional heating of the gas for fast ( $<1 \mathrm{~ms}$ ) switching of the FORT, but no dependence on the magnetic trap switching time or the overlap time.

[24] D. M. Stamper-Kurn, M. R. Andrews, A. P. Chikkatur, S. Inouye, H. -J. Miesner, J. Stenger, and W. Ketterle, Phys. Rev. Lett. 80, 2027 (1998).

[25] We estimate that the variation of the magnetic field across the axial extent of our largest cloud is less than $0.1 \%$.

[26] C. R. Monroe, E. A. Cornell, C. A. Sackett, C. J. Myatt, and C. E. Wieman, Phys. Rev. Lett. 70, 414 (1993).

[27] J. L. Bohn, J. P. Burke, Jr., C. H. Greene, H. Wang, P. L. Gould, and W. C. Stwalley, Phys. Rev. A 59, 3660 (1999); J. P. Burke, Jr., C. H. Greene, J. L. Bohn, H. Wang, P. L. Gould, and W. C. Stwalley, Phys. Rev. A 60, 4417 (1999).

[28] A. Derevianko, W. R. Johnson, M. S. Safronova, and J. F. Babb, Phys. Rev. Lett. 82, 3589 (1999).

[29] H. Wang et al., Phys. Rev. A 62, 052704 (2000).

[30] L. Viverit, S. Giorgini, L. P. Pitaevskii, and S. Stringari, Phys. Rev. A 63, 033603 (2001). 